Fecha de recepción: abril 2021

Fecha de aprobación: mayo 2021

Fecha publicación: junio 2021

\section{Diseño Regenerativo y Economía Circular}

Lorna Lares ${ }^{(1)}$ y Andreé Henríquez ${ }^{(2)}$

Resumen: La humanidad está enfrentando profundos retos a nivel planetario. El cambio climático, el crecimiento de la población y las crecientes tensiones sociales son algunos de los aspectos que, sumados a la pandemia global, están tensionando todos los ámbitos de la vida, en especial en lo referido a la recuperación y regeneración de los ecosistemas.

Es en este contexto, el presente artículo propone una reflexión en torno al vínculo y oportunidades que surgen al integrar los conceptos de Diseño Regenerativo, Economía Circular e Industria 4.0, enfatizando el rol que los diseñadores deben tener en el proceso de producción y consumo de los bienes y servicios para la recuperación y regeneración a nivel global.

Palabras clave: Diseño industrial - Diseño Regenerativo - Economía Circular - Desarrollo Sostenible - Habitar - Sustentabilidad - Cultura Regenerativa.

[Las traducciones de los abstracts fueron supervisadas por el autor de cada artículo 34]

(1) Diseñadora Industrial de la Universidad de Chile. Master of Art in Design Futures. Goldsmiths, University of London, Inglaterra. Estudios en Letras, Universidad Central de Venezuela. Académica del Departamento de Diseño de la Facultad de Arquitectura y Urbanismo y Presidenta del Comité por la Sustentabilidad de la Universidad de Chile. Académica asociada al Observatorio de Innovación, línea diseño y economía circular, de la Facultad de Economía y Negocios de la Universidad de Chile. Investigadora asociada a la Red de Pobreza Energética Chile - línea bienestar, eficiencia energética y contaminación atmosférica. Investigadora asociada al programa transdiciplinario Energía, Agua y Sustentabilidad UChile - línea industria sustentable y economía circular. Área de especialización en metadiseño, sustentabilidad y economía circular. Vasta trayectoria en docencia de diseño y gestión de diseño en instituciones de educación superior. lolares@uchilefau.cl

${ }^{(2)}$ Doctor $\odot$ en Administración y Negocios, Magíster en Administración y Negocios, ambos de la Facultad de Economía y Negocios de la Universidad de Chile. En la actualidad es subdirector del Observatorio de Innovación de la Facultad de Economía y Negocios de la Universidad de Chile, director de investigación del Centro de Innovación y Economía Circular y director del primer Centro Tecnológico de Economía Circular en Chile, consorcio público-privado para fomentar el desarrollo de tecnologías que impulsen la circularidad. Sus áreas de investigación son Economía Circular e Innovación, Transferencia Tecnoló- 
gica Universidad-Industria, e Interdisciplina y Transdisciplina en la Educación Superior. anhenrique@fen.uchile.cl

\section{Introducción}

"Nuestras vidas dependen de la salud del planeta". Es así que el diseño se posiciona como un pilar fundamental para entregar solución a los actuales problemas socioambientales y económicos que están afectando la salud del planeta azul. En ese sentido, la salud del planeta depende de las decisiones de diseño.

La actual crisis sanitaria ha puesto en evidencia la urgencia de establecer una nueva relación con la naturaleza y de repensar el actual modelo de producción y consumo. La economía lineal predominante, "tomar-fabricar-desechar" o "adquirir-usar-eliminar", es responsable en gran medida del agotamiento de los recursos y de la mayor amenaza que ha enfrentado la humanidad, el cambio climático. Todo ello ha puesto en la discusión pública la relevancia de pensar la reactivación de los países desde la mirada del Desarrollo Sostenible. Progresivamente se consolida la idea de que no podemos repetir los errores del pasado, poniendo acento sólo en los indicadores económicos como fuente de la medición del bienestar de las personas y el planeta.

En este contexto, la Economía Circular ha ganado un importante reconocimiento a nivel internacional como un modelo articulador, que permite desacoplar el crecimiento económico del uso de la energía y los materiales, sobre la base de principios restaurativos y regenerativos, donde "el desperdicio es un problema de diseño". Este nuevo modelo requiere, por lo tanto, un fuerte énfasis en innovación en espectros tan amplios como la educación, modelos de negocios, tecnologías, procesos, productos y servicios que presionan por pensar de una forma distinta a la producción tradicionalmente lineal (Merli, Preziosi, \& Acampora, 2018).

Junto con lo anterior, la humanidad está asistiendo a un importante proceso de transformación en la producción y consumo. La industria 4.0, también conocida como la cuarta revolución industrial, está avanzando aceleradamente en todos los campos de la vida. Desde la creación de nuevos materiales, el uso de tecnologías digitales, como la realidad aumentada y la desmaterialización económica, mostrando que estamos asistiendo a cambios profundos que modifican los pensamientos y acciones de usuarios, consumidores y/o clientes, que van desde los productos y servicios a sistemas de alta complejidad como las ciudades (Culot, Nassimbeni, Orzes, \& Sartor, 2020).

Emprendedores, empresas y modelos de negocios que se deben adaptar a estas nuevas transformaciones, diseñan y mejoran sus productos de forma interactiva, colaborativa y conectada con sus clientes, quienes además tienen estilos de vida altamente digitalizados -con una frontera difusa entre vida física y digital. Asimismo, la escala de lo diseñando se ha desplazado, desde el diseño de productos al diseño de nuevos modelos de negocios, empresas, sistemas económicos y el diseño de ciudades circulares. Pasamos de diseñar para un usuario acotado a diseñar para una red de personas intensamente conectadas, que se 
extiende por todo el planeta, tal como lo plantea el diseñador industrial Tim Brown, quien además ha reformulado y reorganizado la metodología de diseño tradicional, hacia una metodología para el desarrollo de un diseño circular. Ya desde 2009, Brown invitaba a los diseñadores a pensar en grande y aseguraba que "el diseño se convirtió en una herramienta del consumismo" (Brown, 2009), con "la Guía del Diseño Circular" entrega una herramienta a los diseñadores para crear modelos de negocios, productos y servicios dentro de un modelo cerrado, alejado del modelo lineal de producción y consumo.

Es en este nuevo escenario, el Diseño Regenerativo surge como una posibilidad para crear soluciones innovadoras, eficientes y ecomiméticas que permitan avanzar hacia una economía circular, pudiendo ser un pilar fundamental para la transformación del actual modelo lineal de producción y consumo en un modelo restaurativo y regenerativo, que equilibre las necesidades de las personas y los ecosistemas, y que posibilite una reactivación económica resiliente y baja en carbono, para afrontar los desafíos globales como el cambio climático, la pérdida de biodiversidad y la posibilidad de vivir en un medio ambiente sano. Se requiere una nueva mentalidad de negocio y un nuevo enfoque de diseño. La Comisión Europea, ha señalado que el $80 \%$ de los impactos ambientales de un producto se define en la etapa de diseño. Sin embargo, el diseño como herramienta y estrategia para la transición a una economía circular no ha ganado mayor relevancia en América Latina. Este desafío implica una mayor y mejor aproximación en las estrategias, las que deben superar la actual gestión de residuos y la escalera de las 3R (reducir, reusar, reciclar). Estrategia y soluciones que solo pueden ser impulsadas a través del diseño regenerativo.

Es esta relación, entre Diseño Regenerativo, Economía Circular e Industria 4.0, que el presente trabajo busca explorar y presentar oportunidades, que emergen para alcanzar los objetivos de recuperación y regeneración de los sistemas socioambientales, donde los diseñadores poseen un rol fundamental. Para lo anterior, en lo que sigue del texto se abordará la idea del Diseño Regenerativo, para posteriormente presentar su vinculo con la Economía Circular y la Industria 4.0, proponiendo en las ultimas dos secciones algunas reflexiones relativas al rediseño de la cultura material y económica imperante.

\section{El futuro del diseño es regenerativo}

En el escenario global actual, complejo y cambiante, el sentido de urgencia en alcanzar la carbono neutralidad, reducir los gases de efecto invernadero, los efectos del cambio climático y reducir el impacto de la actual crisis sanitaria, producida por el COVID-19 (SARS-CoV2), son parte de los desafíos que enfrentamos, no sólo porque afecta la dependencia de los seres humanos sobre los combustibles fósiles sino también por la forma en que vivimos, cómo nos organizamos como sociedad y sus respectivas economías. Kelin (2014) ya hacía énfasis en la necesidad de replantearnos el actual modelo económico y reconstruir las economías locales, o rehacer nuestros sistemas políticos como oportunidad para acortar las enormes desigualdades económicas y sociales.

En este contexto, sin duda que las y los diseñadores han entregado una gran contribución, desde el quehacer disciplinar y profesional. Sin embargo, ha quedado en evidencia que su 
rol ha sido más reactivo que activo. A pesar de las aproximaciones y aportes de los principales enfoques y prácticas de diseño, que consideran los pilares de la sustentabilidad, como el Diseño Sustentable (1980), el Ecodiseño (1990), Diseño para el Medio Ambiente (1992) y el Diseño para la Sustentabilidad (2001), entre otras aproximaciones "verdes", el agotamiento de los recursos, la sobreproducción, la sobreabundancia de desechos y la contaminación ambiental, continúan siendo un problema y un desafío para las futuras prácticas del diseño. Principalmente, porque se han centrado en minimizar los impactos y el uso eficiente de los recursos en post de un desarrollo sostenible sin integrar los problemas complejos que se presentan en los desafíos globales y urgentes.

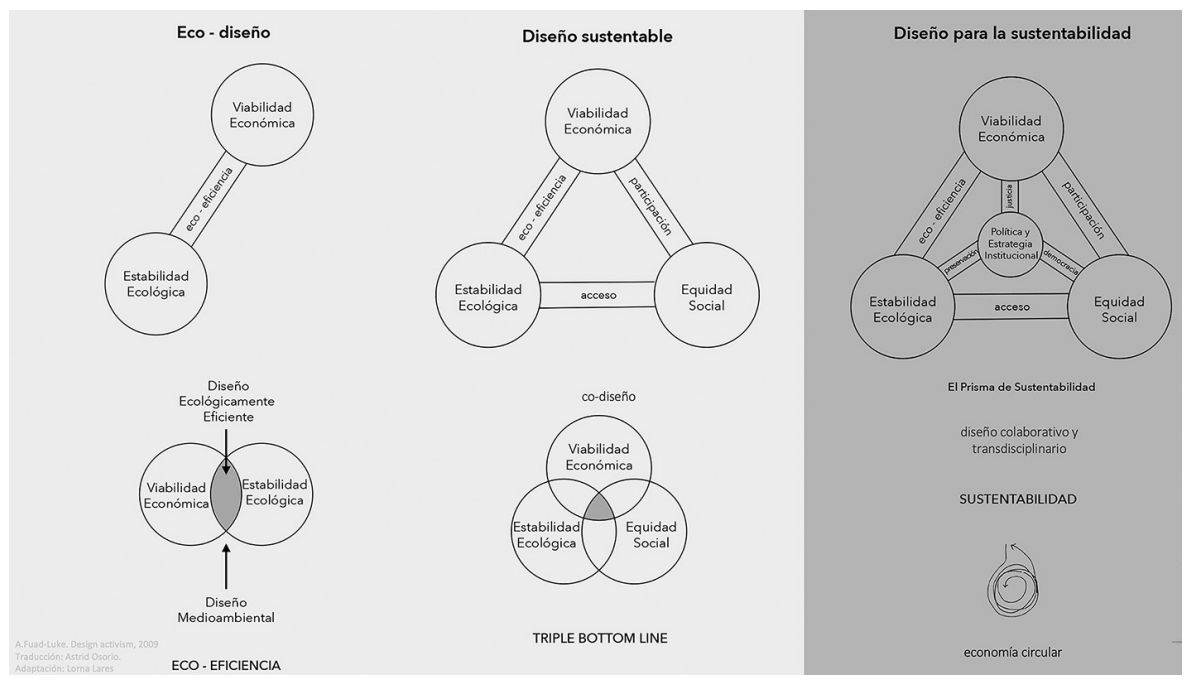

Figura 1. Eco-diseño, Diseño sustentable, Diseño para la sustentabilidad. Fuad-Luke, A. 2009. Adaptación del autor.

Se necesita de un diseño capaz de intervenir en un alto nivel organizacional, de manera holística, transdisciplinaria, adaptativa y eco-mimética, donde el énfasis de su quehacer este centrado en resolver estos problemas complejos y anticipar soluciones hacia un modelo de desarrollo sostenible, basado en una economía regenerativa, con aproximaciones y propuestas integrales, que aporten soluciones en los distintos niveles y dimensiones económicas, políticas, socio ambientales y humanas, capaz de contribuir a las transformaciones estructurales que el planeta y la sociedad en su conjunto necesita. Se necesita entonces de un Diseño Regenerativo. 
Bill Reed (2017) define el Diseño Regenerativo como un sistema de tecnologías y estrategias, basado en la comprensión del funcionamiento interno de los ecosistemas que genera diseños que regeneran conjuntos socio-ecológicos (es decir, generar de nuevo su capacidad inherente de vitalidad, viabilidad y evolución) en lugar de agotar sus sistemas y recursos de soporte vital subyacentes.

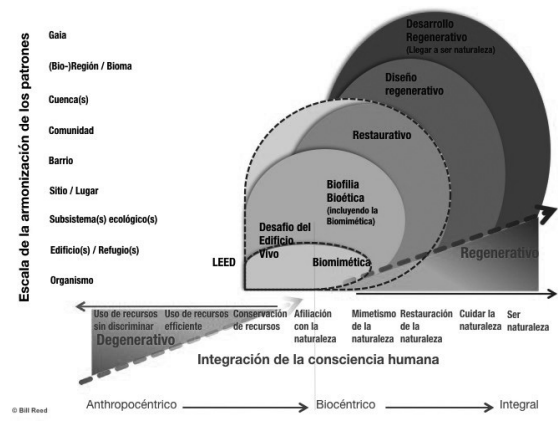

El diseño nos puede ayudar a integrar la riqueza de los conocimientos especializados, las habilidades y aspiraciones compartidas por la humanidad y puede entenderse como la actividad interactiva que conecta las intenciones humanas con su expresión material y cultural en forma de artefactos, instituciones y procesos (Christian, 2016).

Así la velocidad de la revolución tecnológica, la hiperconectividad, la creciente automatización, -acentuada con la actual crisis sanitaria-, y su consecuente impacto multidimensional hace necesario crear un marco de reflexión crítica, sobre la necesidad de una cultura regenerativa de diseño, que aborde el desafío y contribuya a un cambio cultural sistémico que transite (rápidamente) de una mirada antropocentrista hacia una ecocentrista e integral, donde el núcleo humano-naturaleza (diversa y compleja) sea fundamental en este proceso de cambio. Desarrollar nuevas capacidades y especular futuros escenarios -en distintos niveles organizacionales y espacios temporales-, nuevas formas de producción y consumo, de ciudades y de habitar, especialmente en los países de la región, es esencial, debido a que centran su economía en la explotación de commodities y donde una mayor atención en la necesidad de un desarrollo armónico y sustentable es urgente.

En consecuencia, la crisis climática, sanitaria y social, es también una gran oportunidad y una necesidad urgente que requiere sin duda del rol fundamental y activo del diseño, del mismo modo que se requiere de la ciencia y la tecnología. Alcanzar una Reactivación Sostenible para Chile (2020), similar a la iniciativa The Great Reset -El gran reinicio- del Foro 
Económico Mundial (2020), o los objetivos del Pacto Verde de Europa (2019), requieren de una nueva aproximación de diseño que supere las prácticas de diseño verde o centrado en el reciclaje, incluso que vaya más allá del prisma de la sustentabilidad. Una aproximación que posibilite una transformación hacia una cultural regenerativa. Una cultura humana regenerativa es saludable, resiliente y adaptativa, como se muestra en la figura 3.

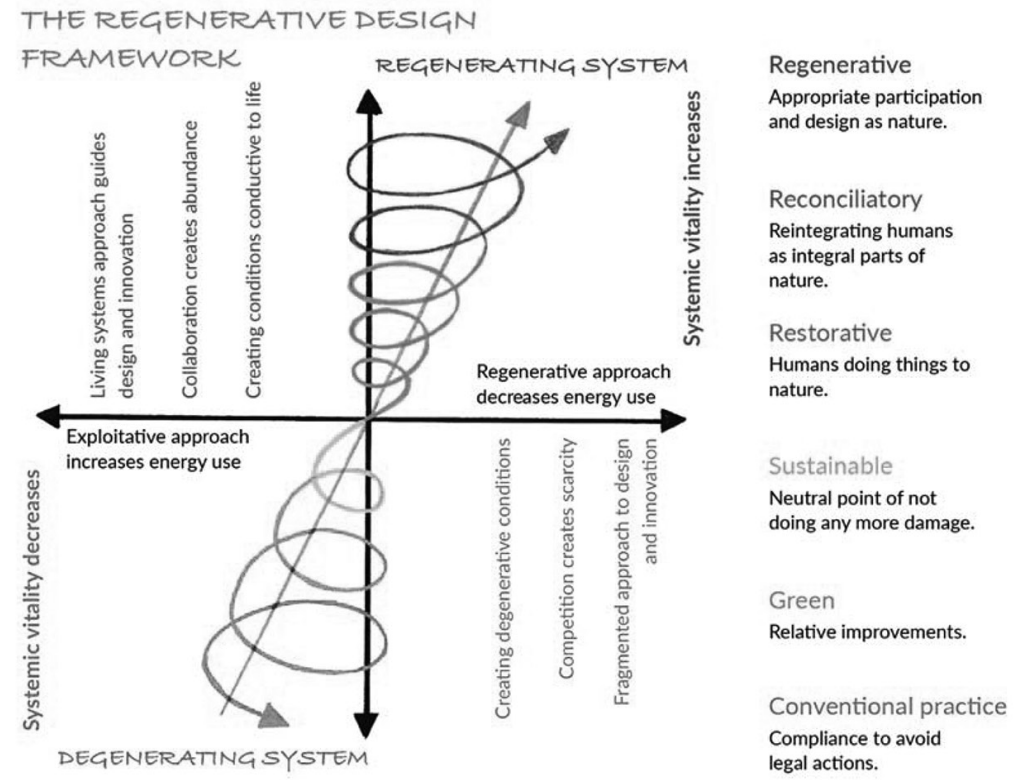

Figura 3. Marco de Diseño Regenerativo. Wahl, D. 2016. Adaptación de Reed, B. 2006.

Ciudades como Finlandia (2016), Londres (2017), Francia (2018) y recientemente Amsterdam (2020), han ocupado un enfoque de diseño holístico, integral y regenerativo, como centro de su estrategia a largo plazo y base para sus políticas públicas, dando a la práctica de diseño un rol fundamental para alcanzar su objetivo de convertirse en ciudades circulares, y crear modelos económicos y energéticos más respetuosos con el medioambiente, con un uso eficiente de los recursos, desarrollo de nuevas alternativas en torno a seguridad alimentaria, arquitectura resiliente, entre otros.

Los productos y servicios que diseñamos, ofrecemos y elegimos como ciudadanos, consumidores o clientes, influyen en la manera como co-creamos y diseñamos la cultura en que 
vivimos. En este sentido, Papanek (1972) afirmaba, sobre el diseño centrado en el sistema económico imperante, que "hay profesiones más dañinas que el diseño industrial, pero muy pocas”. Así mismo, Reed (2017) plantea que el diseño inevitablemente nos instruye sobre nuestra relación con la naturaleza y las personas, nos hace más o menos conscientes y más o menos ecológicamente competentes. El objeto último del diseño no son los artefactos, los edificios o los paisajes, sino las mentes humanas. En el enfoque de economía circular, el diseño se presenta como un pilar fundamental para la transformación del actual modelo lineal de producción y consumo a un modelo de economía restaurativo y regenerativo, que equilibre las necesidades de las personas y los ecosistemas, y que posibilite una reactivación económica resiliente y baja en carbono, para afrontar los desafíos globales como el cambio climático, pérdida de biodiversidad y la posibilidad de vivir en un medio ambiente sano, dando paso a estilos de vidas que resignifiquen el concepto de habitar, en distintas escalas, potenciando el uso de energías, recursos y materiales renovables o que mantengan los nutrientes biológicos e industriales en un mismo bucle o ciclo de uso y/o vida.

Reciclar no es suficiente, si sólo se considera el material (al final del ciclo de uso) como materia prima para otro producto, con menos calidad o de menor complejidad para lo que fue creado, antes de que se convierta en desecho o llegue a vertederos. Aplicar principios de diseño basados en la naturaleza, sean estos a través de la biofabricación de materiales o a través de la biomímesis de sistemas, procesos y/o productos y servicios, es una aproximación que debemos profundizar y continuar desarrollando con mayor consciencia. El enfoque de la Cuna a la Cuna es un ejemplo claro, de cómo puede ser mejor y positivo no solo para el sistema económico sino para todo el ecosistema.

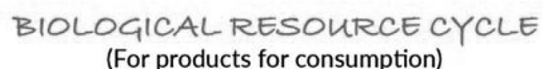

(For products for consumption)

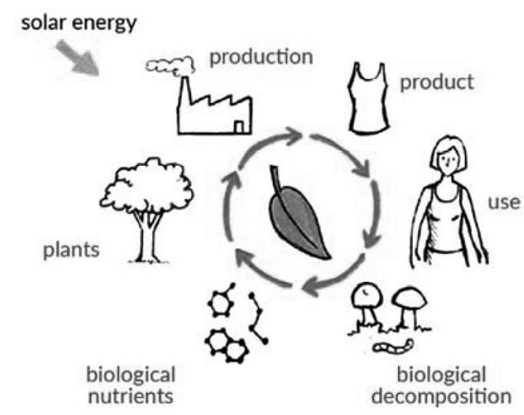

TECHNICAL RESOURCE CYCLE
(For products for service)

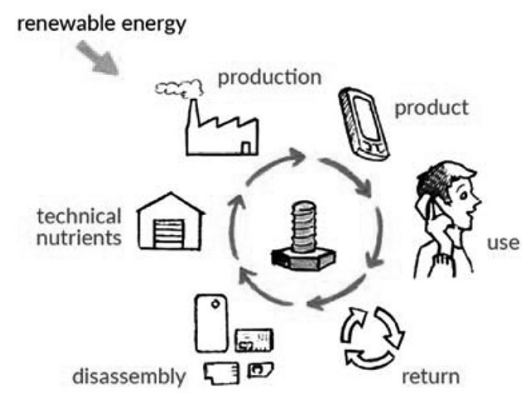

Figura 4. Ciclo de recursos. Wahl, D. 2016. 
Sin embargo, el diseño regenerativo proporciona un marco para crear, aplicar, adaptar e integrar una combinación de tecnologías modernas y antiguas al diseño, la gestión y la continua evolución de los entornos construidos sostenibles, logrando resultados ecológicos y sociales positivos (Reed, 2017). En consecuencia, la interrelación entre innovación, tecnología y diseño regenerativo tienen por objetivo último la salud del sistema integrado, es decir se diseña para los ecosistemas y el planeta. Asumiendo al ser humano y la naturaleza como partes integradas del ecosistema. Aun cuando se pueden integrar distintos enfoques, el marco estructural se centra en la creación de una nueva cultura y en desarrollar capacidades, en distintos niveles y escalas, capaces de sostener y articular las condiciones para la vida de los seres vivos y las comunidades dentro de los límites del planeta, favoreciendo las condiciones para un modelo económico restaurativo y regenerativo, como la economía circular.

\section{La relevancia de incorporar el diseño en la economía circular en la nueva era de la industria 4.0}

La Economía Circular (EC) ha sido promovida por gobiernos, instituciones de cooperación internacional, la academia, empresas y la sociedad civil a nivel global, especialmente en Europa (Korhonen, Honkasalo, \& Seppälä, 2018). Su definición ha sido compleja y no exenta de confusiones en las últimas décadas (Korhonen, Nuur, Feldmann \& Birkie, 2018; Reike, Vermeulen \& Witjes, 2018). De lo anterior ha derivado la necesidad de generar un consenso, sobre todo a nivel de la academia, respecto de lo que se entiende por EC y las posibilidades que entrega. En este punto los trabajos de Prieto-Sandoval, Jaca, \& Ormazabal, (2018), Geisendorf \& Pietrulla (2018) y Kirchherr, Reike, \& Hekkert (2017) han sido fundamentales en entregar claridad y llegar a la conclusión que la EC incorpora dentro del marco conceptual diferentes niveles operacionales desde el reciclaje hasta el rediseño y es resultado, directo e indirecto, de diversos trabajos anteriores en campos como la Economía Azul, Capitalismo Natural, Desempeño Económico, Ecología Industrial, Credle to Cradle, entre otros, que han permitido una base conceptual para su desarrollo actual. Dentro de las múltiples definiciones posibles la de Kirchherr et al. (2017, p. 224) contiene gran parte de los elementos centrales del modelo circular actual:

Una economía circular describe un sistema económico que se basa en modelos comerciales que reemplazan el concepto de 'fin de vida' por reducir, alternativamente reutilizar, reciclar y recuperar materiales en los procesos de producción / distribución y consumo, por lo tanto operando a nivel micro (productos, empresas, consumidores), nivel meso (parques eco-industriales) y nivel macro (ciudad, región, nacional y más allá), con el objetivo de lograr un desarrollo sostenible, lo que implica crear calidad ambiental, económica prosperidad y equidad social, en beneficio de las generaciones actuales y futuras (traducción propia). 
En cuanto a su objetivo, cabe destacar que la economía circular no es un fin en sí mismo, sino un camino para avanzar hacia el reemplazo del modelo lineal que ha imperado hasta ahora (Ellen Macarthur Foundation, 2014). Lo anterior, a partir de ser regenerativa y restaurativa por intención y diseño para permitir que los sistemas cierren sus ciclos en el proceso de generación y captura de valor económico, social y medioambiental, desacoplando el uso de materiales y energía del crecimiento de la producción (Morseletto, 2020). A partir de lo anterior, se han desarrollado múltiples áreas y niveles de conocimiento en economía circular; desde la sistematización y análisis de casos prácticos a través de los estudios de Geng \& Doberstein (2008), el establecimiento de indicadores como los propuestos por Elia, Gnoni, \& Tornese (2017), la importancia de los modelos de negocios con Whalen (2019), la circularidad en ciudad de Prendeville, Cherim, \& Bocken (2018), la innovación e industria 4.0 con Nascimento et al. (2019) y de la siempre esquiva relación de economía circular y comportamiento del consumidor con Hazen, Mollenkopf, \& Wang (2017), entre muchas otras, que explican el 72\% promedio de crecimiento de publicaciones científicas indexadas entre 2015-2020 (Martínez-Cerna, Henríquez-Aravena, \& Freire-Castello, 2019).

Pero desde su origen la Economía Circular siempre ha reconocido la importancia del Diseño para cumplir sus objetivos. Es a partir de la aproximación práctica que posee esta disciplina para pensar y repensar los bienes y servicios, que se abren múltiples oportunidades para impulsar el tránsito desde un modelo lineal hacia uno circular con una mirada sistémica (Mestre \& Cooper, 2017). Si bien la relación entre Diseño y Economía Circular se presenta relativamente nueva en el campo de la investigación, no lo ha sido respecto de sus preocupaciones por el medioambiente y, en algunos casos, por los aspectos sociales. Conceptos como eco-diseño, diseño verde, diseño para el medioambiente o diseño sostenible han emergido en el último tiempo gracias al interés que profesionales e investigadores han desarrollado, para impulsar una producción y consumo más sostenible (Moreno, De los Rios, Rowe, \& Charnley, 2016). Así, el uso de nuevos materiales y procesos (Moorhouse \& Moorhouse, 2017; Muthu \& Savalani, 2019), el diseño modular (Schischke, Proske, Nissen, \& Lang, 2017; Sonego, Echeveste, \& Galvan Debarba, 2018) el desarrollo de herramientas para integrar los aspectos ambientales en el diseño (Bovea \& Pérez-Belis, 2012; Byggeth \& Hochschorner, 2006) o la orientación hacia una producción más limpia (He, Niu, Hou, \& $\mathrm{Li}, 2018$ ) son solo algunos ejemplos que permiten evidenciar la nutrida agenda de investigación en la relación diseño-medioambiente.

Asimismo, la idea de un Diseño Circular también ha encontrado un espacio en este campo, centrándose en dos tipos de estrategias, una para lograr un "bucle de recursos lentos" (slow resource loop) y otra para "cerrar el círculo de recursos" (close resource loop) relacionado con el establecimiento de modelos de negocios circulares (Mestre \& Cooper, 2017). Sin embargo, esta mirada no ha profundizado en alcanzar el objetivo declarado de restauración y regeneración y ha estado fuertemente asociada a Diseño Sostenible y Ecodiseño, donde la metodología de ciclo de vida del producto ha sido su principal herramienta (European Parliament, 2021), dando pasos importantes en materias de reciclaje y reducción de uso de materiales y energía. Es así como Morseletto (2020) ha propuesto la interesante tesis de que la idea de restauración y regeneración han sido raramente abordadas, incluso, desde el marco conceptual de la Economía Circular. 
Es en esta relación de medio y objetivo que el Diseño Regenerativo ha emergido como una nueva oportunidad para retomar la finalidad original de la Economía Circular, más allá de la pura creación y retención de valor en el ciclo de vida del producto y comprender la importancia del capital natural (Cole, 2012). Como establece el provocativo artículo de Kadar \& Kadar (2020) las "sustentabilidad (ya) no es suficiente" cuando en su foco está en "hacer cosas menos malas", rememorando el trabajo de Peter Marcuse que exponía su crítica al concepto de sostenibilidad (Marcuse, 1998, p. 111).

Petrovski, Pauwels, \& González (2021) proponen que el concepto regenerativo significa permitir que los sistemas sociales y ecológicos mantengan un estado saludable y evolucionen. Por su parte, la restauración estaría definida como "el retorno al estado previo u original" (Morseletto, 2020, p. 769). Para el impulso de ambos se requiere guiarse por principios como cero desechos, adaptabilidad, durabilidad, modularidad, re-uso, reciclaje y reducción (Kadar \& Kadar, 2020). Sin esta mirada sistémica, restaurativa y regenerativa en la forma de diseñar productos y servicios la potencialidad de la Economía Circular difícilmente será lograda (Moreno et al., 2016).

Pero esta relación entre EC y DR no se da en el vacío, los profundos procesos de transformación tecnológica y social de la humanidad proponen un nuevo contexto, presente y futuro, donde se desplegarán la Economía Circular y el Diseño Regenerativo. En particular la Cuarta Revolución Industrial (4RI) está impulsando el desarrollo de la próxima generación de tecnología de producción y consumo (Xu, Xu, \& Li, 2018). Esta nueva revolución industrial se caracteriza por el uso a gran escala de las tecnologías de información y comunicación, el desarrollo de nuevos materiales, robotización y electrónica, internet de las cosas, manufactura aditiva y en definitiva la modificación de la cadena de valor completa de los procesos desde el diseño hasta el consumo sobre la base de innovación basada en ciencia (Liao, Deschamps, de Freitas Rocha Loures, \& Ramos, 2017). Este nuevo escenario permite pensar que el futuro de la Economía Circular y el Diseño Regenerativo deban dar cuenta no solo del proceso de transición desde lo lineal a lo circular, sino también desde el átomo hacia el bit, y desde los grandes procesos hacia el nivel molecular que se sintetiza en la idea de los sistemas físico-cibernéticos.

Desde que se anunciara esta nueva era en Hanover 2011, como parte de una nueva política económica para el desarrollo de Alemania sobre la base de ciencia y tecnología (Anand \& Kumar, 2020), la industria 4.0 ha impregnado transversal y verticalmente los diversos procesos de producción y consumo a escala global, permitiendo la interacción e intercambio a nivel de consumidores (C2C), consumidores y máquinas (C2M) y entre máquinas (M2M) (Roblek, Meško, \& Krapež, 2016). Este nuevo contexto apela a una mutación disciplinar en el Diseño en el sentido que proponía Richard Buchanan en 2001, cuando exponía dentro de su idea de estructura de 4 órdenes, la existencia de un cuarto orden referido a sistemas complejos y medioambientales (Buchanan, 2001). Que da sentido a la idea de Dubberly (2008) de moverse desde un ethos orientado hacia sistemas mecánicos hacia uno de sistemas orgánicos.

García Ferrari (2017), logra sintetizar las ideas anteriores al reconocer que la 4RI significa un amalgamiento entre las esferas físicas, digitales y biológicas que significan peligros y oportunidades para la disciplina del Diseño. En particular, la emergencia de un Diseño Regenerativo para impulsar el tránsito desde una economía lineal hacia una circular, se 
construye sobre el ámbito de las oportunidades presentes y futuras de la cuarta revolución. El uso de nuevos materiales, la producción aditiva, las energías renovables, la sensorización, el modelamiento del funcionamiento y efectos de los productos y servicios, gracias al big data, y aprendizaje de máquinas, se conectan con el impulso de nuevos modelos de negocios. Estos modelos de negocios, el producto como servicio, la economía compartida, la simbiosis industrial, revalorización y recuperación de remanufacturación, son ejemplos que se orientan hacia la recuperación y regeneración de los sistemas socio-ambientales a escala global (Bai, Dallasega, Orzes, \& Sarkis, 2020; Han, Heshmati, \& Rashidghalam, 2020; Ramakrishna, Ngowi, Jager, \& Awuzie, 2020; Rossi, Bianchini, \& Guarnieri, 2020; Wang, Umar, Akram, \& Caglar, 2021).

\section{La oportunidad de rediseñar la cultura material y económica}

Esta relación entre Economía Circular, Diseño Regenerativo e Industria 4.0 debe considerarse esencial para alcanzar los objetivos de desarrollo sostenible donde la generación de valor sea económica, social y ambientalmente equilibrada, pero más importante aún la recuperación y regeneración de sistemas. Por una parte, la Economía Circular entrega un marco conceptual que permite hacer converger diversas aproximaciones, que buscan que el cierre de los ciclos biológicos y técnicos, presentes en la producción y consumo de bienes y servicios, sea visto como una alternativa necesaria y viable respecto del modelo lineal de tomar-hacer-desechar. Por su parte, el Diseño Regenerativo es la forma a través de la cual, la EC puede alcanzar sus objetivos, al abordar la necesaria reconceptualización de los bienes y servicios que se consumen y consumirán (Martín, Álvarez, Ordieres-Meré, Villalba-Díez, \& Morales-Alonso, 2021), y la relación de ellos con la naturaleza. Y la Industria 4.0 entrega el contexto sobre el cual la Economía Circular y el Diseño Regenerativo impulsarán sus acciones. En este punto se debe destacar que esta nueva revolución industrial no es en sí misma ni sostenible, circular y/o regenerativa, es ante todo un cambio, como proponía García Ferrari (2017), de las esferas; las esferas físicas, digitales y biológicas para la producción y consumo. Por lo anterior, la EC y DR son las llamadas a dotar de un ethos a este proceso hacia objetivos planetarios urgentes para la sobrevivencia de la especie humana y los ecosistemas.

Lo anterior convoca a repensar la cultura material sobre la cual se han construido las sociedades modernas, la que debe dar paso a un proceso revolucionario, que cambia la base fundamental post revolución industrial. Estas nuevas posibilidades se dan, además, en un momento donde las innovaciones de base tecnológica permiten la entrega de servicios de baja materialidad y trazabilidad sin precedentes y el desarrollo de nuevos materiales, muchos de estos de origen biológico.

Finalmente, la importancia de un bien o servicio no solo está relacionada con su uso momentáneo para satisfacer necesidades, sino con una visión termodinámica ante la cual se requiere pensar desde el diseño el ciclo de uso y de vida completo de lo que la economía entrega y como estos son integrados como parte del sistema natural, donde cultura y naturaleza son parte esencial del sistema. En este sentido, el Diseño Regenerativo no 
solo tiene un rol en el diseño de nuevos bienes y servicios, sino de repensar todo, o gran parte, de lo que ha estado incuestionablemente en uso por más de un siglo y de redefinir o resignificar la cultura. Sin este rol, la Economía Circular no podrá avanzar hacia un cierre de ciclo completo donde materiales y energía se mantengan el mayor tiempo en uso. Por otro lado, nos lleva a reflexionar, cómo en lugar de hacer menos daño al medioambiente aprendemos a participar con el medioambiente en una relación simbiótica de beneficio mutuo, a través del diseño para una cultura regenerativa, ser naturaleza.

\section{Conclusiones y recomendaciones}

En este artículo hemos propuesto una reflexión relativa al vínculo entre Diseño Regenerativo, Economía Circular e Industria 4.0 dada su importancia para avanzar en el cumplimiento de los desafíos a escala planetaria para la recuperación y regeneración de los sistemas. Es evidente que la humanidad transita uno de sus momentos más complejos en su relación con el medioambiente y consigo misma, mostrando la fragilidad de su convivencia.

Creemos necesaria una mirada que nos permita profundizar más en la creación de nuevas estructuras y enfoques, donde el rol del diseño para la transición a una cultura regenerativa, no solo es necesaria sino urgente. La relación tríadica expuesta, abre oportunidades para avanzar, pero implica un desafío para correr las fronteras de la disciplina del diseño, que debe reconocer esta transformación y orientar sus acciones hacia objetivos complejos y en distintos niveles organizacionales. Compartiendo, y adaptando, la idea de Papanek (1973), el diseño puede alcanzar el sitial más relevante, ya que los diseñadores pueden son- ser los responsables de impulsar un nuevo estatus para los productos y servicios que se consumen, los seres humanos, las comunidades, a nivel planetario.

Nuevas investigaciones podrán profundizar en esta relación. En particular se hace necesario abordar el desarrollo de un marco conceptual orientador para operacionalizar el desarrollo conceptual aquí vertido. Además, elementos más específicos como la relación entre los modelos de negocios circulares y Diseño Regenerativo son espacios aún inexplorados en la literatura. Finalmente, la importancia de este tema para los países de América Latina y el Caribe representan otras oportunidades para la investigación y la práctica del diseño.

\section{Referencias}

Anand, N., \& Kumar, A. (2020). A Roadmap to Industry 4.0: Smart Production, Sharp Business and Sustainable Development. (A. Nayyar \& A. Kumar, Eds.), Advances in Science, Technology and Innovation. Cham: Springer International Publishing. https://doi. org/10.1007/978-3-030-14544-6 
Bai, C.; Dallasega, P.; Orzes, G., \& Sarkis, J. (2020). Industry 4.0 technologies assessment: A sustainability perspective. International Journal of Production Economics, 229, 107776. https://doi.org/10.1016/j.ijpe.2020.107776

Bovea, M. D., \& Pérez-Belis, V. (2012). A taxonomy of ecodesign tools for integrating environmental requirements into the product design process. Journal of Cleaner Production, 20(1), 61-71. https://doi.org/10.1016/j.jclepro.2011.07.012

Brown, T. (2009). Change by Design: How Design Thinking Transforms Organizations and Inspires Innovation (First). HarperBusiness.

Buchanan, R. (2001). Design research and the new learning. Design Issues, 17(4), 3-24.

Byggeth, S., \& Hochschorner, E. (2006). Handling trade-offs in Ecodesign tools for sustainable product development and procurement. Journal of Cleaner Production, 14(15-16), 1420-1430. https://doi.org/10.1016/j.jclepro.2005.03.024

Cole, R. J. (2012). Regenerative design and development: Current theory and practice. Building Research and Information, 40 (1), 1-6. https://doi.org/10.1080/09613218.2012.617516

Culot, G.; Nassimbeni, G.; Orzes, G., \& Sartor, M. (2020). Behind the definition of Industry 4.0: Analysis and open questions. International Journal of Production Economics, 226(January), 107617. https://doi.org/10.1016/j.ijpe.2020.107617

Dubberly, H. (2008). Design in the Age of Biology: Shifting From a Mechanical-Object Ethos to an Organic-Systems Ethos. Interactions, 15(5), 35-41. https://doi.org/ $10.1145 / 1390085.1390092$

Elia, V.; Gnoni, M. G., \& Tornese, F. (2017). Measuring circular economy strategies through index methods: A critical analysis. Journal of Cleaner Production, 142(4), 2741-2751. https://doi.org/10.1016/j.jclepro.2016.10.196

Ellen Macarthur Foundation. (2014). Rethink the future.

European Parliament. (2021). ECODESIGN FOR CIRCULAR ECONOMY BEFORE 2017. Retrieved from file://C:/Users/Admin/Desktop/diseño y sustentabilida otras revistas/ new-boost-for-jobs-growth-and-investment_ecodesign-for-circular-economy_202103-01.pdf

García Ferrari, T. (2017). Design and the Fourth Industrial Revolution. Dangers and opportunities for a mutating discipline. Design Journal, 20(sup1), S2625-S2633. https://doi.or g/10.1080/14606925.2017.1352774

Geisendorf, S., \& Pietrulla, F. (2018). The circular economy and circular economic concepts-a literature analysis and redefinition. Thunderbird International Business Review, 60(5), 771-782. https://doi.org/10.1002/tie.21924

Geng, Y., \& Doberstein, B. (2008). Developing the circular economy in China: Challenges and opportunities for achieving "leapfrog development." International Journal of Sustainable Development \& World Ecology, 15(3), 231-239. https://doi.org/10.3843/SusDev.15.3:6

Han, J.; Heshmati, A., \& Rashidghalam, M. (2020). Circular economy business models with a focus on servitization. Sustainability (Switzerland), 12(21), 1-17. https://doi. org/10.3390/su12218799

Hazen, B. T.; Mollenkopf, D. A., \& Wang, Y. (2017). Remanufacturing for the Circular Economy: An Examination of Consumer Switching Behavior. Business Strategy and the Environment, 26(4), 451-464. https://doi.org/10.1002/bse.1929 
He, B.; Niu, Y.; Hou, S., \& Li, F. (2018). Sustainable design from functional domain to physical domain. Journal of Cleaner Production, 197, 1296-1306. https://doi.org/10.1016/j. jclepro.2018.06.249

Kadar, T., \& Kadar, M. (2020). Sustainability Is Not Enough: Towards AI Supported Regenerative Design. Proceedings - 2020 IEEE International Conference on Engineering, Technology and Innovation, ICE/ITMC 2020, 0-5. https://doi.org/10.1109/ICE/ ITMC49519.2020.9198554

Kelin, N. (2014). This Changes Everything. Capitalism vs. Climate (First). Simon \& Schuster. Kirchherr, J.; Reike, D., \& Hekkert, M. (2017). Conceptualizing the circular economy: An analysis of 114 definitions. Resources, Conservation and Recycling, 127(April), 221-232. https://doi.org/10.1016/j.resconrec.2017.09.005

Korhonen, J.; Honkasalo, A., \& Seppälä, J. (2018). Circular Economy: The Concept and its Limitations. Ecological Economics, 143, 37-46. https://doi.org/10.1016/j.ecolecon. 2017.06.041

Korhonen, J.; Nuur, C.; Feldmann, A., \& Birkie, S. E. (2018). Circular economy as an essentially contested concept. Journal of Cleaner Production, 175, 544-552. https://doi. org/10.1016/j.jclepro.2017.12.111

Liao, Y.; Deschamps, F.; de Freitas Rocha Loures, E., \& Ramos, L. F. P. (2017). Past, present and future of Industry 4.0 - a systematic literature review and research agenda proposal. International Journal of Production Research, 55(12), 3609-3629. https://doi.org/10.108 0/00207543.2017.1308576

Marcuse, P. (1998). Sustainability is not enough. Environment and Urbanization, 10(2), 103-112. https://doi.org/10.1177/095624789801000201

Martín, M. G.; Álvarez, A. P.; Ordieres-Meré, J.; Villalba-Díez, J., \& Morales-Alonso, G. (2021). New business models from prescriptive maintenance strategies aligned with sustainable development goals. Sustainability (Switzerland), 13(1), 1-26. https://doi. org/10.3390/su13010216

Martínez-Cerna, L.; Henríquez-Aravena, A., \& Freire-Castello, N. (2019).Economía Circular $y$ políticas públicas. (Konrad-Adenauer-Stiftung e.V.(KAS), Ed.). Konrad-Adenauer-Stiftung e.V. (KAS).

Merli, R.; Preziosi, M., \& Acampora, A. (2018). How do scholars approach the circular economy? A systematic literature review. Journal of Cleaner Production, 178(March), 703-722. https://doi.org/10.1016/j.jclepro.2017.12.112

Mestre, A., \& Cooper, T. (2017). Circular product design. A multiple loops life cycle design approach for the circular economy. Design Journal, 20, S1620-S1635. https://doi.org/10 $.1080 / 14606925.2017 .1352686$

Moorhouse, D., \& Moorhouse, D. (2017). Sustainable Design: Circular Economy in Fashion and Textiles. Design Journal, 20(sup1), S1948-S1959. https://doi.org/10.1080/1460692 5.2017.1352713

Moreno, M.; De los Rios, C.; Rowe, Z., \& Charnley, F. (2016). A conceptual framework for circular design. Sustainability (Switzerland), 8(9). https://doi.org/10.3390/su8090937

Morseletto, P. (2020). Restorative and regenerative: Exploring the concepts in the circular economy. JOURNAL OF INDUSTRIAL ECOLOGY. https://doi.org/10.1111/jiec.12987 
Muthu, S. S., \& Savalani Mahesh, M. (2019). Handbook of Sustainability in Additive Manufacturing - Volume 1 (Vol. 1). Retrieved from http://www.springer.com/series/13340

Nascimento, D. L. M.; Alencastro, V.; Quelhas, O. L. G.; Caiado, R. G. G.; Garza-Reyes, J. A.; Lona, L. R., \& Tortorella, G. (2019). Exploring Industry 4.0 technologies to enable circular economy practices in a manufacturing context: A business model proposal. Journal of Manufacturing Technology Management, 30(3), 607-627. https://doi.org/10.1108/ JMTM-03-2018-0071

Papanek, V. (1973). Design for the Real World: Human Ecology and Social Change (Second). Academy Chicago Publishers; Second.

Petrovski, A. A.; Pauwels, E., \& González, A. G. (2021). Implementing regenerative design principles: A refurbishment case study of the first regenerative building in spain. Sustainability (Switzerland), 13(4), 1-18. https://doi.org/10.3390/su13042411

Prendeville, S.; Cherim, E., \& Bocken, N. (2018). Circular Cities: Mapping Six Cities in Transition. Environmental Innovation and Societal Transitions, 26, 171-194. https://doi. org/10.1016/j.eist.2017.03.002

Prieto-Sandoval, V.; Jaca, C., \& Ormazabal, M. (2018). Towards a consensus on the circular economy. Journal of Cleaner Production, 179, 605-615. https://doi.org/10.1016/j. jclepro.2017.12.224

Ramakrishna, S.; Ngowi, A.; Jager, H. De, \& Awuzie, B. O. (2020). Emerging Industrial Revolution: Symbiosis of Industry 4.0 and Circular Economy: The Role of Universities. Science, Technology and Society, 25(3), 505-525. https://doi.org/10.1177/0971721820912918

Mang, P. \& Reed, B (2017). Regenerative Development and Design 2nd edition. ResearchGate. extraido https://www.researchgate.net/publication/321156684_Update_Regenerative _Development_and_Design_2nd_edition

Reike, D.; Vermeulen, W. J. V., \& Witjes, S. (2018). The circular economy: New or Refurbished as CE 3.0? - Exploring Controversies in the Conceptualization of the Circular Economy through a Focus on History and Resource Value Retention Options. Resources, Conservation and Recycling, 135(February 2017), 246-264. https://doi.org/10.1016/j. resconrec.2017.08.027

Roblek, V.; Meško, M., \& Krapež, A. (2016). A Complex View of Industry 4.0. SAGE Open, 6(2). https://doi.org/10.1177/2158244016653987

Rossi, J.; Bianchini, A., \& Guarnieri, P. (2020). Circular economy model enhanced by intelligent assets from industry 4.0: The proposition of an innovative tool to analyze case studies. Sustainability (Switzerland), 12(17). https://doi.org/10.3390/su12177147

Schischke, K.; Proske, M.; Nissen, N. F., \& Lang, K. D. (2017). Modular products: Smartphone design from a circular economy perspective. 2016 Electronics Goes Green 2016+, EGG 2016, 1-8. https://doi.org/10.1109/EGG.2016.7829810

Sonego, M.; Echeveste, M. E. S., \& Galvan Debarba, H. (2018). The role of modularity in sustainable design: A systematic review. Journal of Cleaner Production, 176, 196-209. https://doi.org/10.1016/j.jclepro.2017.12.106

Wang, K. H.; Umar, M.; Akram, R., \& Caglar, E. (2021). Is technological innovation making world "Greener"? An evidence from changing growth story of China. Technological Forecasting and Social Change, 165(December 2020), 120516. https://doi.org/10.1016/j. techfore.2020.120516 
Whalen, K. A. (2019). Three circular business models that extend product value and their contribution to resource efficiency. Journal of Cleaner Production, 226, 1128-1137. https:// doi.org/10.1016/j.jclepro.2019.03.128

Xu, L. Da; Xu, E. L., \& Li, L. (2018). Industry 4.0: state of the art and future trends. International Journal of Production Research, 56(8), 2941-2962. https://doi.org/10.1080/002 07543.2018.1444806

\begin{abstract}
Humanity is facing profound challenges at the planetary level. Climate change, population growth and increasing social tensions are some of the aspects that, added to the global pandemic, are stressing all areas of life, especially about the recovery and regeneration of ecosystems.

It is in this context that this article proposes a reflection on the link and opportunities that arise when integrating the concepts of Regenerative Design, Circular Economy, and Industry 4.0, emphasizing the role that designers should have in the process of production and consumption of goods and services for the recovery and regeneration at a global level.
\end{abstract}

Keywords: Industrial Design - Regenerative Design - Circular Economy - Sustainable Development - Inhabiting - Sustainability - Regenerative Culture.

Resumo: A humanidade está enfrentando desafios profundos em nível planetário. Mudanças climáticas, crescimento populacional e crescentes tensões sociais são alguns dos aspectos que, somados à pandemia global, vêm afetando todas as áreas da vida, principalmente no que diz respeito à recuperação e regeneração dos ecossistemas.

É neste contexto que este artigo propõe uma reflexão sobre o vínculo e as oportunidades que surgem ao integrar os conceitos de Design Regenerativo, Economia Circular e Indústria 4.0, destacando o papel que os designers devem ter no processo de produção e consumo dos bens e serviços de recuperação e regeneração globalmente.

Palavras chave: Desenho Industrial - Desenho Regenerativo - Economia Circular - Desenvolvimento Sustentável - Habitar - Sustentabilidade - Cultura Regenerativa.

[Las traducciones de los abstracts fueron supervisadas por el autor de cada artículo] 